\title{
High-accuracy wavemeter based on a stabilized diode laser
}

\author{
Ayan Banerjee, Umakant D. Rapol, Ajay Wasan, and Vasant Natarajan ${ }^{\text {a) }}$ \\ Department of Physics, Indian Institute of Science, Bangalore 560 012, India
}

\begin{abstract}
We have built a high-accuracy wavelength meter for tunable lasers using a scanning Michelson interferometer and a reference laser of known wavelength. The reference laser is a frequency-stabilized diode laser locked to an atomic transition in Rb. The wavemeter has a statistical error per measurement of 5 parts in $10^{7}$, which can be reduced considerably by averaging. Using a second stabilized diode laser, we have verified that systematic errors are below 4 parts in $10^{8}$.
\end{abstract}

The use of diode lasers has become very common in the fields of optics and atomic physics. ${ }^{1}$ The principal advantages of these lasers lie in their low cost, narrow spectral width, tunability over several $\mathrm{nm}$, efficient power consumption, reliability, and ease of operation. Single-transversemode laser diodes are available over most of the nearinfrared spectrum from 600-2000 nm. Furthermore, by placing the diode in an external cavity and using optical feedback from an angle-tuned grating, they can be made to operate at single frequency (single longitudinal mode) with linewidths of order $1 \mathrm{MHz}^{2,3}$ Such frequency-stabilized diode lasers have increased the access of researchers to experiments which have previously required expensive ring-cavity dye or Ti-sapphire lasers. In particular, the field of laser cooling has blossomed in the past decade as several alkali atoms and alkali-like ions have cooling transitions in the infrared which are accessible with diode lasers. ${ }^{4}$ However, to use lasers in these applications effectively it is important to know their absolute wavelength accurately. In many cases, this is achieved by locking the laser to a known atomic or molecular transition in a vapor cell. But this may not always be possible, especially when working with short-lived radioactive species or trapped ions. One solution is to measure the wavelength of the laser precisely by beating its frequency against that of a reference laser in a scanning optical interferometer.

In this letter, we present a design for such a wavemeter in which the reference laser is a frequency-stabilized diode laser locked to an atomic transition. This gives the reference laser the desired frequency stability of $1 \mathrm{MHz}$ and the required wavelength calibration for absolute measurements. While expensive commercial wavemeters that use a stabilized $\mathrm{He}-\mathrm{Ne}$ laser as the reference are available, our wavemeter is built in-house around a low-cost laser diode and the entire instrument has a component cost of less than $\$ 1500$. To characterize the accuracy of the instrument, we have measured the wavelength of a second stabilized diode laser system. The results indicate an accuracy of $5 \times 10^{-7}$ with a $25 \mathrm{~cm}$ scan distance, and that systematic errors are below $4 \times 10^{-8}$.

The wavemeter, shown schematically in Fig. 1, is based on a design first reported by Hall and Lee. ${ }^{5}$ The basic idea is

${ }^{a)}$ Electronic mail: vasant@ physics.iisc.ernet.in to obtain the wavelength of an unknown laser in terms of the wavelength of the reference laser using a scanning Michelson interferometer where both lasers traverse essentially the same path. As the interferometer is scanned, the interference pattern on the detectors goes alternately through bright and dark fringes. Since both lasers traverse the same path, the ratio of the number of fringes counted after scanning through a certain distance is the ratio of the two wavelengths. The ratio obtained is a wavelength ratio in air, however, the wavelength ratio in vacuum (or equivalent frequency ratio) is easily calculated by making a small correction for the dispersion of air $^{6}$ between the two wavelengths. Thus, if the absolute wavelength of the reference laser is known, the wavelength of the unknown laser can be determined.
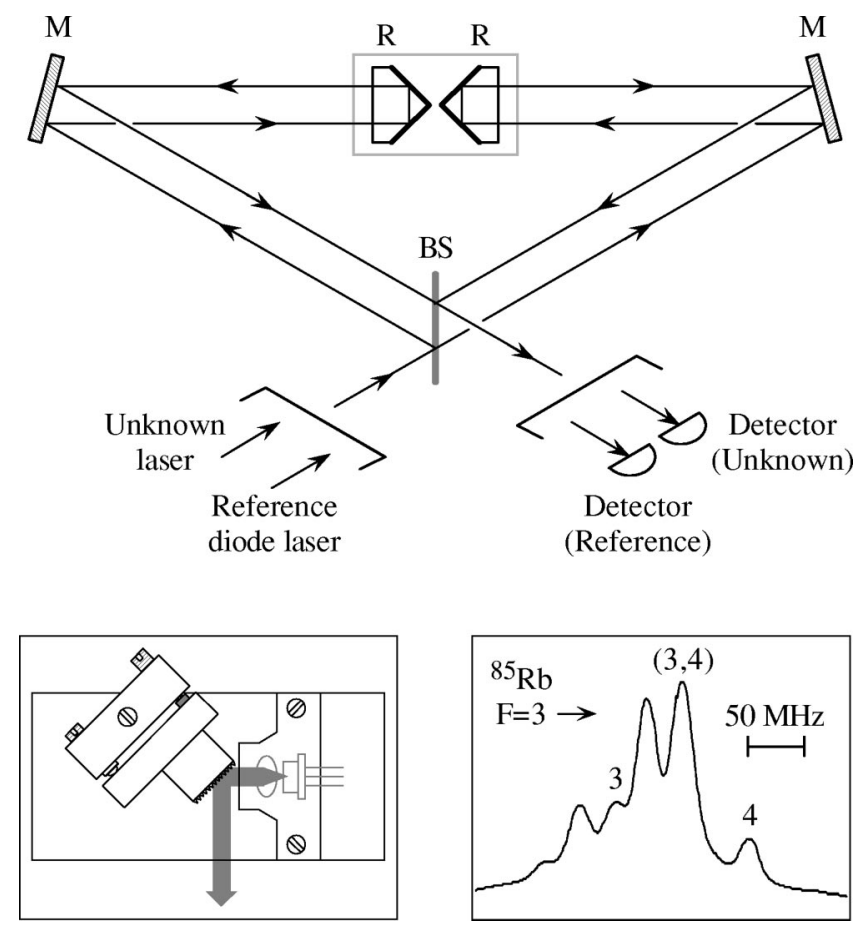

FIG. 1. Schematic of the wavemeter. The wavemeter is a scanning Michelson interferometer consisting of a beamsplitter (BS), two end mirrors (M), and two retroreflectors (R). The retroreflectors are mounted back to back on a movable cart. The inset on the bottom left shows the assembly of the reference diode laser with collimating lens and piezomounted grating. The inset on the right is a Doppler-free saturated-absorption spectrum in ${ }^{85} \mathrm{Rb}$ as the laser is scanned across hyperfine transitions starting from the $F=3$ level. The laser is locked to the $(3,4)$ crossover peak corresponding to a frequency of $3.8422958 \times 10^{14} \mathrm{~Hz}$. 
The interferometer consists of a beamsplitter, two end mirrors, and two retroreflectors mounted back to back on a movable cart. The retroreflectors, made of three mutually orthogonal mirrors, ensure that the return beams are displaced from the input beams and do not feed back into the laser. The movable cart is a brass block riding on a pressurized air bearing, which allows it to slide freely over a distance of about $100 \mathrm{~cm}$. The push-pull design of the interferometer, with the two retroreflectors mounted back to back, has the advantage that the interference pattern goes through a complete fringe cycle for every $\lambda / 4$ displacement of the cart, a factor of 2 improvement over designs where only one arm of the interferometer is scanned. The interferometer produces two output beams; of these the one on the opposite side of the beamsplitter from the input beam has near-perfect contrast $(>90 \%$, in our case) because it is a combination of beams each of which is once reflected and once transmitted through the beamsplitter. ${ }^{7}$ This beam is detected for both the reference laser and the unknown laser by Si PIN photodiodes and the two signals fed into a frequency counter. The counter has a ratio function and directly displays the ratio of the two inputs with user-settable integration times of up to $10 \mathrm{~s}$.

The diode laser system used as the reference laser is built around a commercial single-mode laser diode (Mitsubishi ML60125R-01) with a nominal operating wavelength of $785 \mathrm{~nm}$ and $\mathrm{cw}$ output power of $30 \mathrm{~mW}$. The light is collimated using a $4.5 \mathrm{~mm}, 0.55 \mathrm{NA}$ aspheric lens. The laser is frequency stabilized in a standard external cavity design (Littrow configuration) ${ }^{2}$ using optical feedback from an 1800 lines/mm diffraction grating mounted on a piezoelectric transducer (see the inset of Fig. 1). Using a combination of temperature and current control, the diode is tuned close to the $780 \mathrm{~nm} D_{2}$ line in atomic $\mathrm{Rb}\left(5 S_{1 / 2} \leftrightarrow 5 P_{3 / 2}\right.$ transition). A part of the output beam is tapped for Doppler-free saturatedabsorption spectroscopy in a $\mathrm{Rb}$ vapor cell. ${ }^{2}$ The various hyperfine transitions in the two common isotopes of $\mathrm{Rb}$ $\left({ }^{85} \mathrm{Rb}\right.$ and $\left.{ }^{87} \mathrm{Rb}\right)$ are clearly resolved, as shown in the inset of Fig. 1. The linewidth of the hyperfine peaks is $15-20 \mathrm{MHz}$; this is somewhat larger than the $6.1 \mathrm{MHz}$ natural linewidth and is primarily limited by power broadening due to the pump beam. ${ }^{8}$ The injection current into the laser diode is modulated slightly to obtain an error signal and the laser is locked to the $F^{\prime}=(3,4)$ crossover resonance in ${ }^{85} \mathrm{Rb}$, i.e., 60 $\mathrm{MHz}$ below the $F=3 \leftrightarrow F^{\prime}=4$ transition. From the Rb energy level tables ${ }^{9}$ and measured hyperfine shifts, ${ }^{10}$ this corresponds to a frequency of $3.8422958 \times 10^{14} \mathrm{~Hz}$. The elliptic laser beam $\left(5.8 \mathrm{~mm} \times 1.8 \mathrm{~mm} 1 / e^{2}\right.$ diam $)$ is directly fed into the interferometer. The large Rayleigh ranges $(\sim 34$ and $\sim 3 \mathrm{~m}$, respectively) ensure that the beam remains collimated over the length of the interferometer.

To characterize the wavemeter, we have measured the wavelength of a second identical diode laser system but which was locked to a different hyperfine transition in ${ }^{85} \mathrm{Rb}$. The measurement serves two purposes: first, the scatter in the data gives an estimate of the statistical error associated with our instrument since both the reference laser and the unknown laser are stabilized to linewidths below $1 \mathrm{MHz}$ (about 3 parts in $10^{9}$ ), and second, the data tell us if there are any systematic errors associated with our instrument because the difference between the two laser frequencies is already

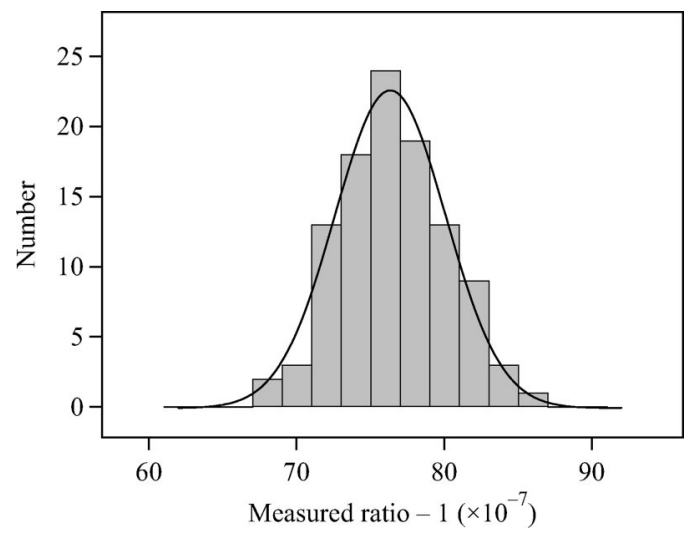

FIG. 2. Histogram of measured ratios. The graph is a histogram of the ratios measured with the second laser locked to a hyperfine transition of ${ }^{85} \mathrm{Rb}$ that is $2944 \mathrm{MHz}$ away from the reference laser. The solid line is a Gaussian fit to the histogram, which yields a mean value of $1.00000763(4)$ and a spread of $5.3 \times 10^{-7}$. The mean corresponds to a frequency difference of 2932(16) $\mathrm{MHz}$, showing that any systematic errors are below $16 \mathrm{MHz}$.

known very precisely. ${ }^{10}$ The measurements were done with the second laser locked to a hyperfine transition in ${ }^{85} \mathrm{Rb}$ that was $2944 \mathrm{MHz}$ higher than the frequency of the reference laser. This implies that the measured ratio should be 1.000007 66. The actual values obtained are shown in Fig. 2 as a histogram plot. The data have a fairly good Gaussian distribution and the fit yields a mean value of $1.00000763(4)$ and a spread of $5.3 \times 10^{-7}$.

Statistical errors in the measured data arise mainly because the frequency counter only detects zero crossings and does not count fractional fringes. The total number of fringes counted depends on the fringe rate (or cart speed) coupled with the $10 \mathrm{~s}$ integration time. Currently, our photodiode electronics limits the cart speed so that we can use only about $25 \mathrm{~cm}$ of cart travel per measurement. This results in the single-shot statistical error of 5 parts in $10^{7}$ in the data. ${ }^{11}$ However, the mean value has an error of only $4 \times 10^{-8}$ since it is an average of more than 100 individual measurements. With some improvements in the counting electronics, it should be possible to use $50-100 \mathrm{~cm}$ of cart travel for each measurement and thereby reduce the statistical error per measurement below $1 \times 10^{-7}$. Resolution enhancement is also possible by phase locking to the fringe signal, as described in Ref. 5. This allows fractional fringes to be counted accurately and the statistical error to be reduced for the same cart travel. Finally, data contamination can occur if partial fringes are counted when the cart turns around at the end of its travel. We, therefore, take data only when the cart is traveling in one direction.

It is important to eliminate all sources of systematic error when aiming for such high accuracy. The chief cause of systematic error is nonparallelism of the two beams in the interferometer. Any misalignment would cause an increase in the measured ratio given by $1 / \cos \theta$, where $\theta$ is the angle between the beams. We have tried to minimize this error by using the unused output beam of the reference laser (the one on the same side of the beamsplitter as the input beam) as a tracer for aligning the unknown laser beam, and checking for parallelism over a distance of about $2 \mathrm{~m} .{ }^{12}$ The consistency of the mean value of $1.00000763(3)$ with the expected value (1.000 00766$)$ shows that this method works quite well for 
accuracies up to $4 \times 10^{-8}$. We have also found it useful to check for parallelism by looking for a minimum in the measured ratio as the angle of the unknown beam is varied. This works because the measured value is always larger than the correct value, whether $\theta$ is positive or negative, and becomes minimum when $\theta=0$.

In conclusion, we have built a high-accuracy wavemeter using a scanning Michelson interferometer and a reference diode laser. The frequency-stabilized diode laser is locked to an atomic transition in $\mathrm{Rb}$ for absolute calibration. Using a second stabilized diode laser, we have shown that the statistical error in each measurement is 5 parts in $10^{7}$, and the systematic error is less than 4 parts in $10^{8}$. We have recently used this wavemeter to measure the wavelength of a diode laser tuned to the $795 \mathrm{~nm} D_{1}$ line in $\mathrm{Rb}\left(5 S_{1 / 2} \leftrightarrow 5 P_{1 / 2}\right.$ transition), ${ }^{13}$ which yields a precise value for the finestructure interval in the $5 P$ state of $\mathrm{Rb}$. The precision obtained is an order of magnitude better than published values ${ }^{9}$ and demonstrates the power of the instrument for precision spectroscopy experiments. Hyperfine splittings, which are of order $\mathrm{GHz}$, are more accessible to techniques such as microwave resonance or heterodyne measurements. ${ }^{14}$ On the other hand, fine-structure splittings are of order $\mathrm{THz}$ and our technique is uniquely suited for precise measurements in this range of frequency differences.

The authors are grateful to S. Navaneetha for machining the mechanical components of the wavemeter. This work was supported by research grants from the Board of Research in Nuclear Sciences (DAE), and the Department of Science and Technology, Government of India. A.B. and A.W. acknowledge financial support from CSIR.
${ }^{1}$ C. E. Wieman and L. Hollberg, Rev. Sci. Instrum. 62, 1 (1991).

${ }^{2}$ K. B. MacAdam, A. Steinbach, and C. Wieman, Am. J. Phys. 60, 1098 (1992).

${ }^{3}$ L. Ricci, M. Weidemüller, T. Esslinger, A. Hemmerich, C. Zimmermann, V. Vuletic, W. König, and T. W. Hänsch, Opt. Commun. 117, 541 (1995).

${ }^{4} \mathrm{~A}$ good review of laser cooling experiments is contained in the Nobel Prize lectures: S. Chu, Rev. Mod. Phys. 70, 685 (1998); C. N. CohenTannoudji, ibid. 70, 707 (1998); W. D. Phillips, ibid. 70, 721 (1998).

${ }^{5}$ J. L. Hall and S. A. Lee, Appl. Phys. Lett. 29, 367 (1976); a wavelength meter based on this design was made commercially available by NIST in the 1980s.

${ }^{6}$ B. Edlén, Metrologia 2, 71 (1966).

${ }^{7}$ We obtain better than $90 \%$ fringe contrast even though our beamsplitter is close to 60/40. The other output beam has perfect contrast only if the beamsplitter is exactly $50 / 50$. However, as discussed later, this beam is useful in reducing systematic errors by ensuring that the unknown beam is parallel to the reference.

${ }^{8}$ Collisional broadening at the gas pressure in the $\mathrm{Rb}$ vapor cell is estimated to be $\sim 10 \mathrm{kHz}$ and the linewidth of the laser has been independently measured to be $\sim 1 \mathrm{MHz}$.

${ }^{9}$ C. E. Moore, Natl. Stand. Ref. Data Ser. 35, 180 (1971).

${ }^{10}$ E. Arimondo, M. Inguscio, and P. Violino, Rev. Mod. Phys. 49, 31 (1977).

${ }^{11}$ The calculated error from missing one fringe over a distance of $25 \mathrm{~cm}$ is about $8 \times 10^{-7}$ at $780 \mathrm{~nm}$. The measured error is smaller because we are measuring a wavelength ratio very close to 1 , and chances are high that either both counters see a zero crossing or miss it. In fact, if we try to measure a ratio of exactly 1 by locking both lasers to the same transition, we get a bimodal distribution in the measured ratios: 1.0000000 most of the time and occasionally 1.0000008 , which corresponds to one extra fringe counted by the second detector. That is why we have chosen to measure a test ratio of 1.00000766 where the distribution is Gaussian.

${ }^{12}$ Ideally, the unknown beam should enter exactly where the reference beam exits so that both beams traverse exactly the same path in opposite directions. However, this causes one laser to feed into the other and destabilize it. Therefore, we align the beams with a small offset and check for parallelism over a finite distance.

${ }^{13}$ A. Banerjee, U. D. Rapol, and V. Natarajan (unpublished).

${ }^{14}$ J. Ye, S. Swartz, P. Jungner, and J. L. Hall, Opt. Lett. 21, 1280 (1996). 